\title{
Software review
}

\section{Auto-ID technology in retail and its potential application in marketing}

Received: 25th January, 2004

\section{Shaun Doyle}

is CEO of Cognitive Box, a marketing technology solution provider. Previously he was VP Intelligent Marketing Solutions at SAS. In this role he worked with various parts of the SAS organisation to develop business-orientated solutions for marketing, in particular the SAS Marketing Automation (MA) Solution and SAS's industry-specific solutions for telco and retail banking. He was founder and chairman of Intrinsic, a campaign management vendor acquired by SAS in March 2001 . He is well known in the marketing industry and regularly participates in leading industry conferences. He is also author of a number of papers on the application of database marketing that have been published in the UK and elsewhere.

Shaun Doyle

Cognitive Box (Consulting) Ltd, Sams Barn,

The Greenway,

West Hendred, Wantage, Oxon, OX12 8RD, UK.

Tel: +44 (0)1235 821353 ; Fax: +44 (0)1235 861678 ; e-mail: shaun.doyle@ cognitivebox.com

Abstract This paper describes the Auto-ID technology and its key components. It explores some of the issues associated with its widespread use. Finally it explores its potential business applications in marketing.

\section{INTRODUCTION}

The Auto-ID Center at Massachusetts Institute of Technology (MIT) ${ }^{1}$ announced the launch of version 1.0 of the EPCglobal Network in September 2003. This was a key milestone in the launch of a global set of standards and technologies that allow individual items to be tagged with microchips or radio frequency identification (RFID) tags. These tags carry the electronic product code (EPC), which allows these objects to be uniquely identified and, through wireless technology, detailed information to be maintained on the object. Thus, the products on the shelf can not only talk to you, they have a distributed memory.

This paper describes the Auto-ID technology and its key components and explores some of the issues associated with its widespread use. Finally it discusses the potential business applications in marketing.

\section{AUTO-ID TECHNOLOGY}

The primary focus of the Auto-ID technology is to embed the EPC into product items; this results in objects that are intelligent and can communicate.

There are several components that make up Auto-ID technology. ${ }^{2}$ These include:

- eTag, an electronic tag

- EPC, a unique identifier

- object name service (ONS)

- Savant systems

- physical markup language (PML)

- business applications

\section{The EPC}

The EPC is a corner stone of Auto-ID technology. It is a string of numbers that provides a unique identification; for instance, instead of referring to a class of products - as universal product codes 
(UPCs) do - the EPC refers to a specific instance of a product.

\section{eTag}

To facilitate Auto-ID, the EPC is embedded in a memory chip contained within a smart tag (eTag) on individual products. The chip in turn is connected to an antenna. This allows the eTag to be scanned by a radio frequency reader, which transmits the embedded identity code of the product to a network, where the detailed information on the product is kept. This detailed information can then be communicated from the network to provide whatever information is necessary about that product.

RFID is the basis for current Auto-ID technology. It is important to note that the baseline functionality of these eTags provides read-only access to the EPC. No detailed information need be kept on the eTag.

The current technology and standards do not preclude other tags with read-write functionality or even more advanced capabilities. However, as additional functions and capabilities increase, so will the cost of eTags. At the present time, read-write tags tend to be slower and have a shorter range than their read-only counterparts.

The implementation of EPC does not depend on RFID technology; any way of quickly and easily reading a unique ID from a product will work. RFID is the most common option at the moment, but other technologies are being tested.

\section{The ONS}

The next link in the Auto-ID chain is the ONS. The ONS tells computer systems where to find information about any object that carries an EPC.
ONS is based in part on the internet's existing Domain Name System (DNS), which routes information to appropriate network interfaces. The ONS will likely be many times larger than the DNS, serving as a lightning fast 'post office' that locates data for the trillions of objects that will eventually carry an EPC.

\section{Savant systems}

Savant is a software technology that acts as the 'central nervous system' of the EPCglobal Network. Savant manages and moves information in a way that does not overload existing corporate and public networks.

\section{PML}

PML is a new standard language for describing physical objects, in the same way that hypertext markup language (HTML) is the common language on which most internet web pages are based. The PML describes the physical characteristics of an object and almost anything can be contained within the description, such as weight or calorific content, repair instructions and audit trails. PML will allow manufacturers and retailers to specify and customise the information tracked on products. (It is also technically possible for the consumer to start to collect information on the objects that they own.)

There will not be a vast repository of PML descriptions. Ultimate implementation of the PML descriptions will result in highly distributed data. Manufacturers, retailers and consumers will all have unique views to data.

\section{Business applications}

Potential application for Auto-ID in business are numerous. They include: 
- manufacturing process control

- inventory management

- supply chain optimisation

- regulatory compliance

- recall management and recycling.

In all these areas, Auto-ID offers the potential for significant savings, as well as new sources of incremental revenue. New services will start to emerge as objects start to become smart and interactive. As the technology becomes pervasive, benefits will extend throughout the entire value chain and for the consumer.

Auto-ID technology has the capability to redefine the global marketplace by embedding intelligence, identity and internet connectivity into everyday objects. The EPC unites elements of the entire supply chain, making it an interactive, dynamic cycle from raw material and distribution to point-of-purchase and recycling, and back to raw material. Products equipped with smart tags will interact with manufacturers, their trading partners and each other to form an optimally efficient cycle of direct, real-time supply and demand.

\section{APPLICATIONS IN MARKETING}

The primary focus of Auto-ID applications has been the supply chain, where it is believed the highest benefits will come. But Auto-ID and similar technologies have a number of unique features that could provide value for marketing. These include the ability to:

- uniquely identify an object

- integrate data from a wide range of sources

- read the EPC wirelessly

- provide communication during the product purchase decision
- access the consumer after a purchase is made.

These features allow the marketer to explore a number of new activities. Many of these can be grouped under the concept of a 'personal shopping assistant'. Examples of these applications in the store and in the home are discussed below.

\section{In the store}

Select an item and view product attributes such as:

- where it was made

- how long it has been in the store

- its expiry date

- what the calorific value is

- what it contains.

Select an item and

- remotely look up the contents of the home larder and confirm if the item is required, alerting the customer where appropriate

- alert customer about products to which they may be allergic, eg contains peanuts

- alert customer that they have a discount voucher for an item.

Select an item and view usage information, such as:

- a recipe for an item

- product survey information

- product instructions

- comparison of prices at other stores.

Prompt customer with location of item:

- pre-defined shopping lists can warn customer when they are in the proximity of a required item 
- a selected recipe can warn the customer when they are in proximity of the required item.

Select an item and receive a promotional offer:

- provide the customer with a promotional offer at the purchase decision point based on the current contents of their basket

- provide the customer with a promotional offer at the purchase decision point based on previous purchase behaviour.

\section{In the home}

Automated shopping lists can:

- use information about items in household and consumption patterns to automatically create a shopping list for a particular store

- use information about pricing to optimise shopping based on price comparisons.

Select an item and:

- warn if an item has gone past its expiry date

- alert customer to a product to which a member of their household may be allergic, eg contains peanuts.

This is just a short list of potential marketing applications; the key point is that this technology will allow us to integrate data from a wide range of sources wirelessly.

\section{ISSUES WITH AUTO-ID}

As with any revolutionary technology, there will be challenges to overcome in Auto-ID implementation. Some challenges are technological in nature, some economic, and some societal. Issues include:

- privacy

- accuracy

- interference

- performance

- frequency availability

- security

- data ownership.

These are discussed in more detail below.

\section{Privacy}

Perhaps the most controversial issue is that of privacy. The ability to track an item after it has been purchased raises a number concerns for consumers. Although there are limits to the current technology, in the future it may be possible to trace clothing stolen from a store for example. If this then gets sold on to an individual who visits a location with a radio receiver, the system could then 'check' to see if it had been paid for.

As consumers see value in the technology, and if these genuine privacy concerns are addressed by the industry, acceptance is likely to increase.

There are also some legitimate competitive issues that come under the heading of privacy. For instance, since the EPCs will be global and unique, it may be possible to determine specific product information from the EPC given enough data. Imagine gaining knowledge of your competitors' shelf assortment and inventory levels by walking through a store accompanied by a hand-held reader.

\section{Accuracy}

Readers cannot be guaranteed to be able to communicate with all tags in a volume all of the time. Environmental issues, the 
make-up of the products being tagged and the volume of tags to be read all impact on read accuracies. The degree of concern is proportional to how much an enterprise relies on absolute data. RFID offers many advantages over manual or semi-automated data collection processes. Any shortcomings in accuracy can be mitigated through the use of redundant readers, information auditing and process redesign.

\section{Interference}

As readers proliferate, more occurrences of interference will be seen. Depending on the frequencies and powers used, devices such as mobile phones, wireless handsets and industrial equipment may be affected. As such a widespread penetration of radio frequency (RF) technology has not been undertaken before, it is difficult to state categorically what will be impacted.

The perceived health risks of this many RFs may also be a concern. While there is no evidence that there are any negative effects at the power and frequency levels associated with RFID, it has not yet been rolled out on such a large-scale. More research and monitoring will need to be conducted to address the public's concerns in this matter.

\section{Performance}

Smart objects could generate tremendous amounts of data. This much data will not be accessible if stored in a massive central repository, so some distribution of data will be necessary. This raises a number of performance issues.

Auto-ID is based on RFID technology. Anyone who has used a mobile phone will be aware of the issues associated with access to a network. In order to work therefore, the data associated with EPCs will need to be available on demand, anywhere.

\section{Frequency availability}

Since RFID currently uses sections of the unlicensed RF spectrum, the available parts of the spectrum that are usable for RFID are an issue. Although there are some frequencies that are common, there is no universal standard. $13.56 \mathrm{MHz}$ and $2.45 \mathrm{GHz}$ are both worldwide standard industrial, scientific and medical (ISM) frequencies. These are available in most parts of the world, albeit at slightly different regulations.

More useful in terms of read range and speeds are tags operating at roughly $915 \mathrm{MHz}$, or ultra-high frequency (UHF). The UHF spectrum around 900 $\mathrm{MHz}$ is not universally available at the same frequency and power levels worldwide, however.

This issue will be addressed through two potential methods. The first alternative is multi-frequency readers overall RF system design (integration of antenna, readers and tags) is the most difficult part of the problem here. The second is to select a common frequency. Obviously, since this involves millions of stakeholders, the lead-time on this will be considerable. This does not, however, deal with the fact that not all frequencies work well for every application (although some work well across virtually all applications).

\section{Security}

Security will be paramount and may be viewed at a number of levels, including:

- read security (or being able to read the tag)

- security of the data

- other security issues. 
For users of the technology to feel comfortable, there will need to be assurances that no one will be able to 'hack' into a smart object. As long as tags are read-only and are difficult to counterfeit, then security will be high. Users of Auto-ID technology will also need to rely on the security of Auto-ID data on the network.

\section{Data ownership}

Related to security, data ownership is an issue. Who owns the massive amounts of event information associated with an object?

It is clear that the manufacturer owns the design specifications and other PML-type data for a given product. It is clear who owns captured data - the owner of the reader that reads the tag. It is less clear, however, how information will be shared.

Many parties will be privy to, and will update, the data for an object as it passes though a supply chain. Will those collecting the data ever want to share data? Does an end-user (consumer) ultimately own a product and its data and, if so, how does use of that data for process improvement or data mining impact privacy?

Lastly, although killing a tag when purchased has been discussed as an option, this method eliminates future recycling benefits. It also introduces the potential for tags to be killed maliciously or by accident, before they should be.

\section{CONCLUSIONS}

The ability to uniquely identify an item through the use of an EPC is a natural extension of the UPC. Allowing this EPC to be wirelessly read and integrated with detailed data across a global network is a major leap in functionality, which will provide manufacturers, retailers and consumers with significant benefits.

The development of Auto-ID technology is evolving, but widespread use is unlikely for many years. The early adopters are likely to be industries where the value of a unit is high and tracking individual items is important. Prime targets include pharmaceuticals and the automotive sectors.

To date, the focus has been on improving supply chain management. Little attempt has been made to explore the potential marketing applications of this technology. The author believes that it has a number of unique features that will prove valuable to marketers when developing point of sale communications. It also offers the opportunity to extend the marketing communication process into the home environment and acess the full product lifecycle.

\section{Acknowledgments}

Auto-ID, eTag, EPCglobal Network and Savant System are registered trademarks of the Auto-ID Center, all rights reserved.

\section{References and notes}

1 Auto-ID Center, Massachusetts Institute of Technology, 77 Massachusetts Avenue, Building 3-449, Cambridge, MA, USA.

2 Chappell, G., Ginsburg, L., Schmidt, P., Smith, J. and Tobolski, J. (2002) 'Auto-ID on Demand: The Value of Auto-ID Technology in Consumer Packaged Goods Demand Planning, published by Auto-ID, sponsored by Accenture, 11th November.

\section{Further reading}

Gramling, K., Bigornia, A. and Gilliam, T. (2003) 'FPC Forum', 2nd January.

'Globesity'. Forum for Consumer Products and Retail Leadership, Vol. 5, No. 4, Q4, 2003. 